\title{
Comparison between 3-dimensional cranial ultrasonography and conventional 2-dimensional cranial ultrasonography in neonates: impact on reinterpretation
}

Yu Jin Kim ${ }^{1,2}$, Young Hun Choi ${ }^{2,3}$, Hyun Hae Cho ${ }^{4}$, So Mi Lee ${ }^{5}$, Ji Eun Park ${ }^{2}$, Jung-Eun Cheon ${ }^{2,3}$, Woo Sun Kim, ${ }^{2,3}$ In-One Kim

${ }^{1}$ Department of Radiology, Seoul National University Hospital, Seoul; ' 2 Department of Radiology, Chungbuk National University Hospital, Cheongju; ${ }^{3}$ Department of Radiology, Seoul National University College of Medicine, Seoul; ${ }^{4}$ Department of Radiology, Ewha Womans University Mokdong Hospital, Seoul; ${ }^{5}$ Department of Radiology, Kyungpook National University Hospital, Daegu, Korea

Purpose: The aim of this study was to evaluate impact of 3-dimensional cranial ultrasonography (3DUS) on reinterpretation of cranial ultrasonography images in neonates in comparison with 2-dimensional cranial ultrasonography (2DUS).

Methods: We retrospectively enrolled 50 young infants who simultaneously underwent both 2DUS and 3DUS scanning from February to March 2015. Two pediatric radiologists independently reviewed both scans for overall image quality on a 5-point scale. Five features were evaluated in both scans: the presence of germinal matrix hemorrhage (GMH), intraventricular hemorrhage (IVH), ventriculomegaly (VM), abnormality of periventricular echogenicity (PVE), and focal parenchymal lesions (FL). The concordance rate between the two scanning modes was calculated. The confidence level for each finding on a 3-point scale and the scanning time were compared between the two scanning modes. Interobserver agreement was evaluated using kappa statistics. Results: Both scans demonstrated similar overall image quality in terms of reinterpretation (mean scores for 2DUS and 3DUS, 4.0 0.5 and $4.0 \pm 0.7$ in reviewer $1,3.9 \pm 0.6$ and $4.0 \pm 0.8$ in reviewer 2, respectively). GMH, IVH, VM, and FL showed perfect concordance, while PVE showed a concordance rate of $91.4 \%$ between the two modes by both reviewers. 3DUS was associated with a higher diagnostic confidence in the evaluation of GMH, IVH, and $F L$ than 2DUS $(P<0.05)$ for both reviewers. For PVE, 3DUS received a significantly higher confidence score than 2DUS from one of the reviewers. The mean scanning time for 2DUS and 3DUS was 92.75 seconds and 36 seconds, respectively. Interobserver agreement for qualitative scoring was almost perfect.

Conclusion: In reinterpretation, 3DUS showed very high concordance with 2DUS and a similar image quality. 3DUS also increased diagnostic confidence for several image findings and significantly decreased scan time.

Keywords: 3-Dimensional ultrasound; Infant, newborn; Infant; Ultrasonography; Brain

Received: February 7, 2017

Revised: May 25, 2017

Accepted: May 28, 2017

Correspondence to:

Young Hun Choi, MD, Department of Radiology, Seoul National University Hospital, 101 Daehak-ro, Jongno-gu, Seoul 03080, Korea

Tel. +82-2-2072-3608

Fax. +82-2-747-5781

E-mail: iater@snu.ac.kr

This is an Open Access article distributed under the terms of the Creative Commons Attribution NonCommercial License (http://creativecommons.org/ licenses/by-nc/3.0/) which permits unrestricted noncommercial use, distribution, and reproduction in any medium, provided the original work is properly cited.

Copyright (C) 2018 Korean Society of Ultrasound in Medicine (KSUM)

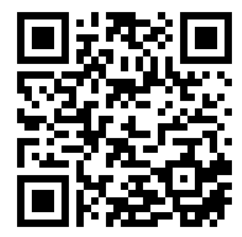

How to cite this article: Kim YJ, Choi YH, Cho HH, Lee SM, Park JE, Cheon JE, et al. Comparison between 3-dimensional cranial ultrasonography and conventional 2-dimensional cranial ultrasonography in neonates: impact on reinterpretation. Ultrasonography. 2018 Jan;37(1):63-70. 


\section{Introduction}

Neonatal cranial ultrasonography (US) is a primary, non-sedative, and widely used image screening tool for the evaluation of various intracranial abnormalities such as hemorrhage or parenchymal abnormalities, hydrocephalus, and congenital malformations $[1,2]$. However, the use of conventional 2-dimensional (2D) cranial ultrasonography (2DUS) has several limitations: it is laborintensive, inefficient, operator-dependent, time-consuming, and involves the difficult task of simultaneous scanning and diagnosis. In addition, other radiologists who do not carry out the scans personally may encounter difficulties in achieving diagnostic confidence and accuracy using previously captured sonograms of the brain $[3,4]$. In our country, Korea, radiologists perform cranial US themselves, taking a standard set of images. In this scenario, the reinterpretation of US examinations by other radiologists can prove to be difficult and unconvincing, making it difficult to build an effective consultation system, although standard views are provided [5]. Nevertheless, expert opinions or reinterpretations from fellow consultants are frequently required in medical practice [3].

Recent advances in US technology have introduced 3-dimensional (3D) cranial US (3DUS), which acquires complete data sets within the volume of interest using various methods: free-hand acquisition with a tracking system, acquisition with a motor-driven 3D transducer, and acquisition with a 2D matrix array transducer [6], thereby allowing a comprehensive understanding of anatomical structures and disease entities, as well as the reconstruction of other planes [7]. Publications have acknowledged the success of 3DUS in the fields of obstetrics and cardiology and the diagnosis of prostate and breast disorders $[2,5,7-9]$. Furthermore, the 3DUS technique can enable the almost complete and consistent acquisition of volume data without missing regions, which in turn may improve the diagnostic confidence of interpreters, thereby overcoming the limitations of 2DUS [10]. Therefore, the purpose of this study was to evaluate the usefulness of 3DUS in the reinterpretation of cranial sonograms when compared with those obtained from 2DUS.

\section{Materials and Methods}

\section{Patients}

Our institutional review board approved this retrospective study and the requirement for informed patient consent was waived. From February to March 2015, 50 infants who had undergone both 2DUS and 3DUS scans in the same examination session were included in our study. Twenty-six infants were male and 24 were female, and the average gestational age at birth was 30 weeks and 2 days (range, 23 weeks to 41 weeks and 2 days). The mean age of infants at the time of cranial US was 35.1 days (range, 3 to 157 days). Among the 50 infants included in the study, eight underwent US scans twice, and therefore, a total of 58 pairs of 2DUS and 3DUS scans were analyzed.

Twenty-nine patients showed normal cranial US results, while the remaining 21 patients recorded the following brain abnormalities according to their medical records, including prior and followup imaging studies such as US and magnetic resonance imaging (MRI): hemorrhage $(n=12)$, white matter injury of prematurity $(n=7)$, hemorrhagic infarction $(n=2)$, congenital bilateral perisylvian polymicrogyria $(n=1)$, and other conditions, including mega cisterna magna $(n=1)$, extra-axial space widening along the frontoparietal convexity $(n=2)$, and choroid plexus cyst $(n=1)$. Comorbid abnormalities included intraventricular hemorrhage (IVH) and white matter injury of prematurity $(n=3)$, germinal matrix hemorrhage $(\mathrm{GMH})$ and hemorrhagic infarction $(n=2)$, and IVH and posthemorrhagic hydrocephalus $(n=1)$.

\section{US Techniques}

US was performed using a single US machine (Logiq E9, GE Healthcare, Waukesha, WI, USA) with a RNA5-9-D broad-spectrum real-time $4 \mathrm{D}$ micro-convex transducer (bandwidth range, 3.0 to 10.0 MHz) and a 9L-D broad-spectrum linear transducer (bandwidth range, 2.0 to $9.0 \mathrm{MHz}$ ). Three pediatric radiologists (S.M.L., H.H.C., and Y.H.C., with 6, 6, and 11 years of clinical experience, respectively) obtained all cranial sonograms in this study. Our routine $2 \mathrm{D}$ cranial US protocol includes obtaining coronal and sagittal static images of the cerebrum through the anterior fontanelle and transverse images of the cerebellum via the mastoid fontanelle. The coronal images include a minimum of six coronal imaging planes at the level of the frontal horn of the lateral ventricle (anterior to the foramen of Monro), the foramen of Monro, the posterior third ventricle and the thalamus, the quadrigeminal cistern, the trigone of the lateral ventricle, and the parieto-occipital periventricular white matter. The sagittal images include a minimum of five images at the midline, the bilateral angled parasagittal plane, and the bilateral tangential parasagittal plane $[1,11,12]$.

The 3DUS scanning was performed using a RNA5-9-D broadspectrum real-time $4 \mathrm{D}$ micro-convex transducer (bandwidth range, 3.0 to $10.0 \mathrm{MHz}$ ). This $3 \mathrm{D}$ probe mechanically covers $116^{\circ}$ and acquires the volume data in one sweep $[8,10,13]$. Mechanical cine sweeps were acquired twice in the coronal and sagittal directions through the anterior fontanelle. The posterior third ventricle and the thalamus were the center of the volume scan in the coronal plane, and the corpus callosum was the center of the volume scan in the sagittal plane. In our study, the volume scan was obtained in the static 3D acquisition mode with $116^{\circ}$ in width and $90^{\circ}$ in the sweep 
range of the volume of interest at 2.2 seconds per sweep (47 frames per second).

\section{Imaging Analysis}

Two pediatric radiologists (K.Y.J. and C.Y.H., with 4 and 11 years of clinical experience, respectively) independently assessed both the 2DUS and 3DUS scans on the same picture archiving and communicating system (INFINITT PACS, INFINITT Healthcare, Seoul, Korea). The reviewing radiologists were blinded to subject information and the prior interpretation. The 2DUS and 3DUS images were evaluated in two distinct sessions in order to prevent recall bias. The overall subjective image quality was rated on both scans on a 5-point scale: 5 points, excellent; 4 points, good; 3 points, average; 2 points, poor; and 1 point, very poor. Abnormal images were reviewed for five findings: $\mathrm{GMH}$, IVH, ventriculomegaly, abnormality of periventricular echogenicity (PVE), and focal parenchymal lesions. The IVH reading was divided into two grades: IVH occupying less than $50 \%$ of the area of the lateral ventricles (group 1) and IVH occupying more than 50\% of the area of the lateral ventricles (group 2). Ventriculomegaly was defined as a widened anterior horn of the lateral ventricle, measuring over 10 $\mathrm{mm}$ on the coronal image at the level of the foramen of Monro. PVE was evaluated in the parieto-occipital periventricular white matter and classified as normal, borderline, or abnormal. PVE was defined as normal if it was homogenous and less echogenic than the choroid plexus [14]. The echogenicity of the periventricular white matter was considered abnormal if it was higher than that of the choroid plexus and heterogeneous, with or without hyperechoic foci [14]. Focal parenchymal lesions included any focal lesion in the brain parenchyma, such as cystic periventricular leukomalacia, lenticulostriate vasculopathy, or a focal infarction. The diagnostic confidence for each of the findings was described on a 3-point scale: 3 points, definite; 2 points, probable; and 1 point, suspicious. The reviewers created the reference standards for the presence and the degree of lesions jointly, by reviewing the available medical records, including prior and follow-up imaging studies. Interobserver agreement in the scoring of the qualitative image analysis was evaluated.

\section{Acquisition Time}

Scanning times were calculated for the 2DUS and 3DUS examinations. The scanning times for the 2DUS and 3DUS examinations were calculated as the time between the first and the last images being taken in each coronal and sagittal scan. The single-sweep scanning time for 3DUS was fixed as 2.2 seconds.

\section{Statistical Analysis}

The concordance rate between findings observed on the 2DUS and 3DUS scans was calculated. The diagnostic confidence of each finding and the overall image quality was compared between 2DUS

Table 1. Results of cranial ultrasonography and concordance rates of the imaging analysis

\begin{tabular}{|c|c|c|c|c|c|c|c|c|c|}
\hline & & \multicolumn{3}{|c|}{ Reviewer $1^{\text {a) }}$} & \multicolumn{3}{|c|}{ Reviewer $2^{\text {a) }}$} & \multicolumn{2}{|c|}{ Kappa value } \\
\hline & & 2DUS & 3DUS & $\begin{array}{l}\text { Concordance rate between } \\
\text { 2DUS and 3DUS (\%) }\end{array}$ & 2DUS & 3DUS & $\begin{array}{l}\text { Concordance rate between } \\
\text { 2DUS and 3DUS (\%) }\end{array}$ & 2DUS & 3DUS \\
\hline \multirow[t]{2}{*}{ GMH } & Present & 13 & 13 & 100 & 13 & 13 & 100 & 1 & 1 \\
\hline & Absent & 45 & 45 & & 45 & 45 & & & \\
\hline \multirow[t]{4}{*}{ IVH } & Present & & & 100 & & & 100 & 1 & 1 \\
\hline & Grade 1 & 3 & 3 & & 3 & 3 & & & \\
\hline & Grade 2 & 1 & 1 & & 1 & 1 & & & \\
\hline & Absent & 54 & 54 & & 54 & 54 & & & \\
\hline \multirow[t]{3}{*}{ PVE } & Normal & 42 & 42 & 91.4 & 34 & 35 & 91.4 & 0.83 & 0.86 \\
\hline & Borderline & 8 & 9 & & 16 & 15 & & & \\
\hline & Abnormal & 8 & 7 & & 8 & 8 & & & \\
\hline \multirow[t]{2}{*}{ VM } & Present & 2 & 2 & 100 & 2 & 2 & 100 & 1 & 1 \\
\hline & Absent & 56 & 56 & & 56 & 56 & & & \\
\hline \multirow[t]{2}{*}{$\mathrm{FL}$} & Present & 5 & 5 & 100 & 5 & 5 & 100 & 1 & 1 \\
\hline & Absent & 53 & 53 & & 53 & 53 & & & \\
\hline
\end{tabular}

2DUS, 2-dimensional cranial ultrasonography; 3DUS, 3-dimensional cranial ultrasonography; GMH, germinal matrix hemorrhage; IVH, intraventricular hemorrhage; PVE, periventricular echogenicity; VM, ventriculomegaly; $F L$, focal parenchymal lesion.

${ }^{\text {a) }}$ Almost perfect interobserver agreement was found (kappa value $>0.8$ ) for each parameter using kappa statistics. 
and 3DUS using the Wilcoxon signed-rank test. The weighted Cohen kappa test was used to evaluate the interobserver agreement in the scoring of the qualitative image analysis. A kappa value $\leq 0.20$ indicated slight agreement; $0.21-0.40$, fair agreement; $0.41-0.60$, moderate agreement; $0.61-0.80$, substantial agreement; and 0.81 0.99 , almost perfect agreement [15]. The acquisition times for 2DUS and 3DUS were compared using the paired t test. All statistical analyses were performed using SPSS ver. 20.0 (IBM Corp., Armonk, NY, USA). P-values of $<0.05$ were considered to indicate statistical significance.

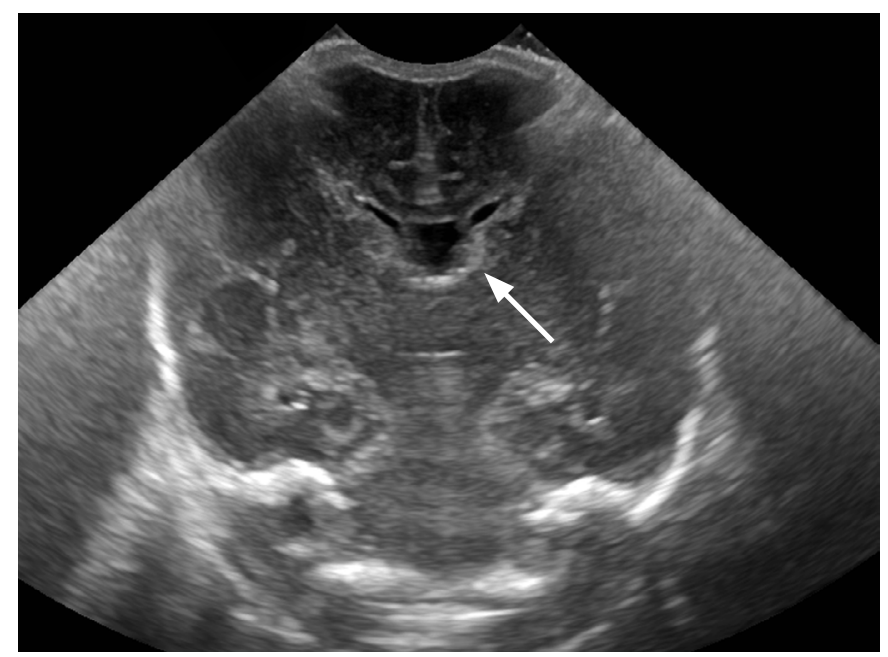

A

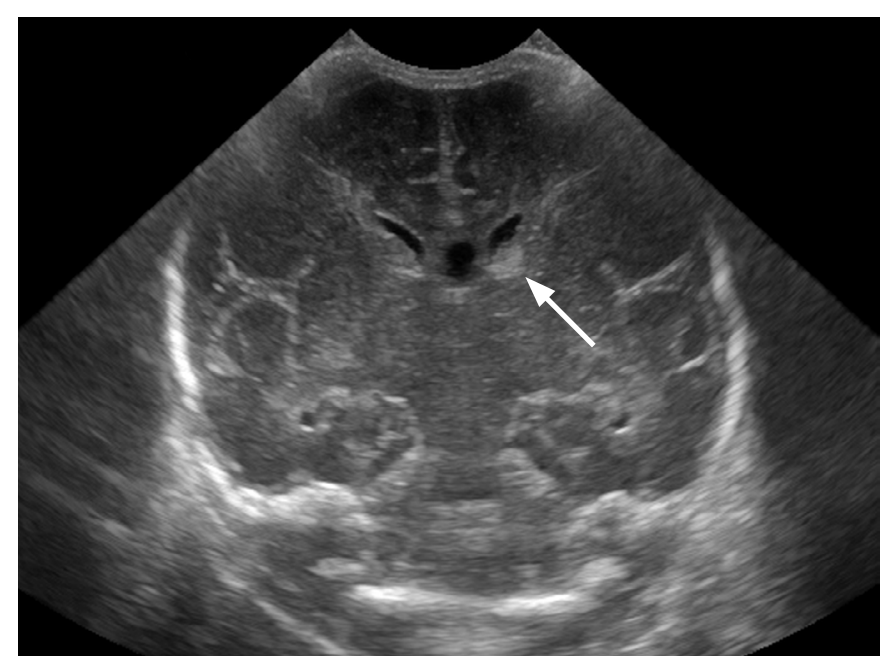

C

Fig. 1. A 30-day-old preterm infant with germinal matrix hemorrhage (GMH) and abnormal periventricular white matter.

A-D. The 2-dimensional cranial ultrasonography (2DUS) images (A, B) and cranial 3-dimensional cranial ultrasonography (3DUS) captured images (C, D) are shown. Left small GMH (arrows in A and C) was noted on both 2DUS (A) and 3DUS (C). Heterogeneous periventricular white matter echo at left frontoparietal white matter (arrows in B and D) is shown at 2DUS images (B) and 3DUS captured images (D). This is one discordant case on 3DUS between the two reviewers; reviewer 1 scored abnormal periventricular echogenicity (PVE) on 2DUS but borderline PVE on 3DUS, reviewer 2 scored abnormal PVE on both 2DUS and 3DUS. It was later diagnosed as a non-cavitary white matter injury on follow-up magnetic resonance imaging. The cine clip of 3DUS scan is presented as Video clip 1. 
lesions were 100\% between 2DUS and 3DUS for both reviewers. A relatively low concordance rate of $91.4 \%$ between 2DUS and 3DUS was noted for PVE by both reviewers. All 13 cases of GMH were demonstrated on both 2DUS and 3DUS, and were successfully screened by both reviewers (Fig. 1, Video clip 1). An IVH group 1 finding was recorded in three patients, and IVH group 2 was recorded for one patient (Fig. 2, Video clip 2). All IVH cases were graded equally on both 2DUS and 3DUS by both reviewers. The findings in patients who showed GMH and IVH on both 2DUS and 3DUS were consistent with the reference standards. In the case of $P V E$, reviewer 1 reported abnormal PVE in eight patients on 2DUS and in seven patients on 3DUS. Reviewer 2 reported abnormal PVE in the same eight patients on both 2DUS and 3DUS (Table 2). Therefore, one discordant case on 3DUS was noted between the two reviewers, in which reviewers 1 and 2 scored the case as borderline and abnormal PVE on 3DUS, respectively; it was later diagnosed as a non-cavitary white matter injury on follow-up MRI (Fig. 1, Video clip 1). The focal lesions, including cystic white matter injury in two patients, a hemorrhagic infarction in one patient, lenticulostriate vasculopathy in one patient, and a hemorrhagic infarction with lenticulostriate vasculopathy in one patient, were assessed as the same on 2DUS and 3DUS by both reviewers (Fig. 3, Video clip 3). In the diagnostic confidence analysis, both reviewers reported an average confidence level in the imaging analysis of "probable" or above (range of mean values, 2.00 to 3.00) for both 2DUS and 3DUS (Table 3). Furthermore, 3DUS was associated with a significantly higher confidence score in the evaluation of $\mathrm{GMH}$, $I V H$, and focal lesions for both reviewers. For the evaluation of $P V E$, the confidence level difference between 2DUS and 3DUS was statistically nonsignificant for reviewer 1 , while reviewer 2 gave a significantly higher confidence score for 3DUS than for 2DUS. The weighted Cohen kappa values in the assessment of inter-reviewer agreement was almost perfect (range, 0.83 to 1) between the two radiologists. The mean scanning time for 2DUS was 92 seconds (range, 13 to 312 seconds). The mean scanning time for 3DUS was 36 seconds (range, 22 to 54 seconds). 3DUS scanning times were

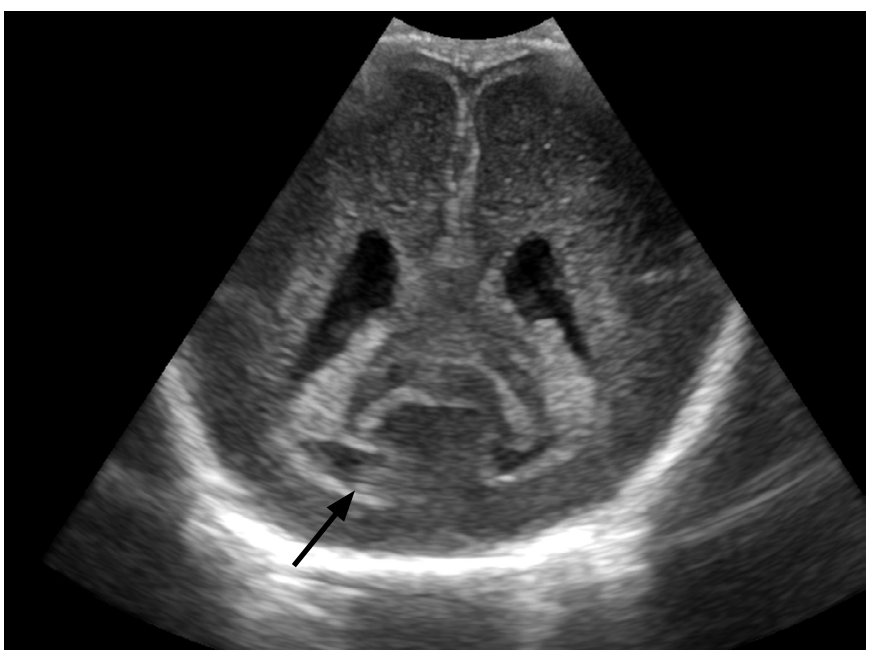

A

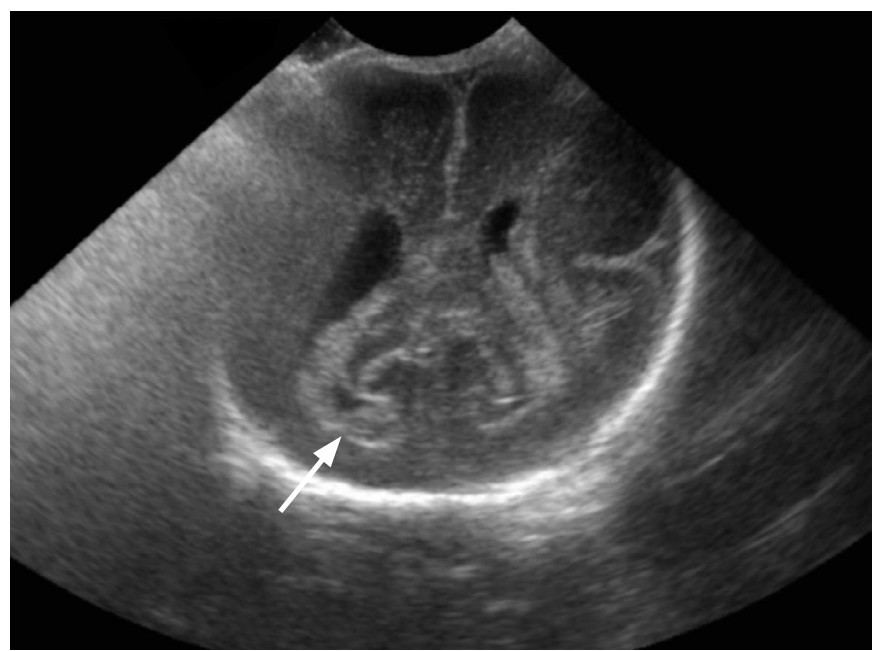

B

Fig. 2. A 34-day-old preterm infant with intraventricular hemorrhage (IVH).

A, B. The 2-dimensional cranial ultrasonography (2DUS) images (A) and cranial 3-dimensional cranial ultrasonography (3DUS) captured images (B) are shown. Small IVH in right lateral ventricle (arrow in A) is suspected on 2DUS. The 3DUS scan shows small IVH (arrow in B) in right lateral ventricle clearly more than 2DUS. The cine clip of 3DUS scan is presented as Video clip 2.

Table 2. Comparison of the score distribution in PVE evaluations on 2DUS and 3DUS by the reviewers

\begin{tabular}{|c|c|c|c|c|c|c|c|}
\hline & \multirow{2}{*}{ PVE } & \multicolumn{3}{|c|}{ Reviewer 1 3DUS } & \multicolumn{3}{|c|}{ Reviewer 2 3DUS } \\
\hline & & Normal & Borderline & Abnormal & Normal & Borderline & Abnorma \\
\hline \multirow[t]{3}{*}{ 2DUS } & Normal & 40 & 2 & 0 & 32 & 2 & 0 \\
\hline & Borderline & 2 & 6 & 0 & 3 & 13 & 0 \\
\hline & Abnormal & 0 & 1 & 7 & 0 & 0 & 8 \\
\hline
\end{tabular}

PVE, periventricular echogenicity; 2DUS, 2-dimensional cranial ultrasonography; 3DUS, 3-dimensional cranial ultrasonography. 


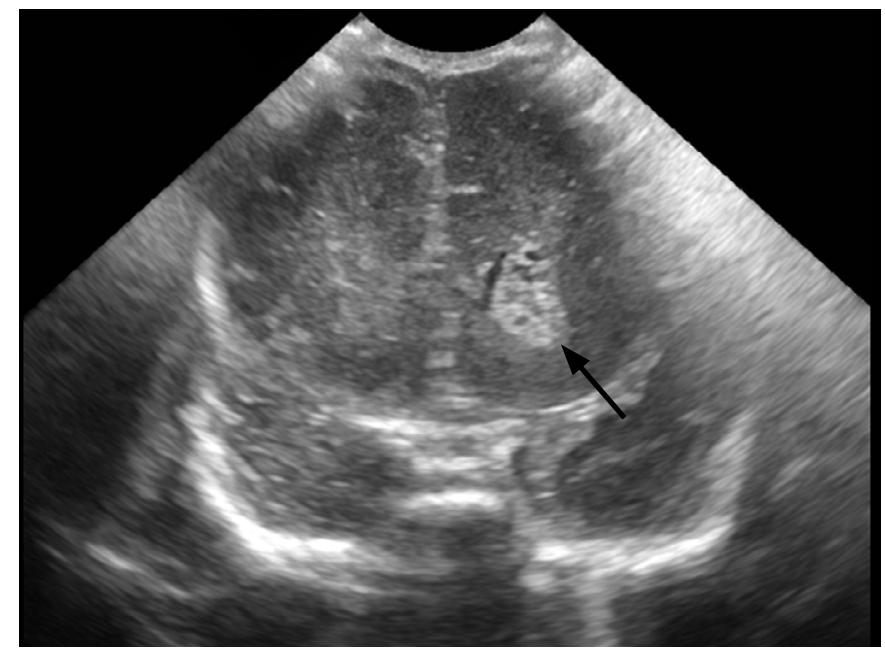

A

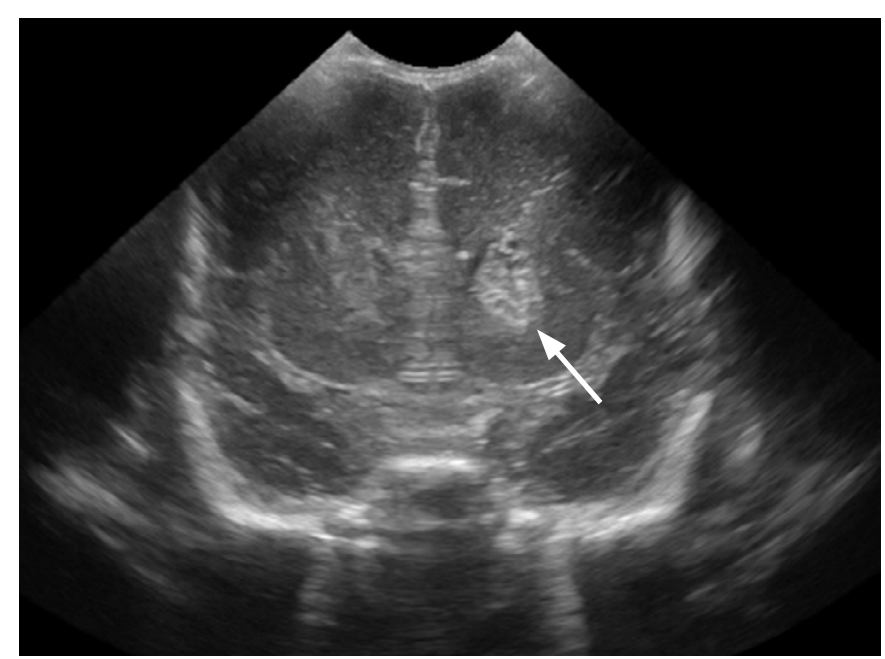

C

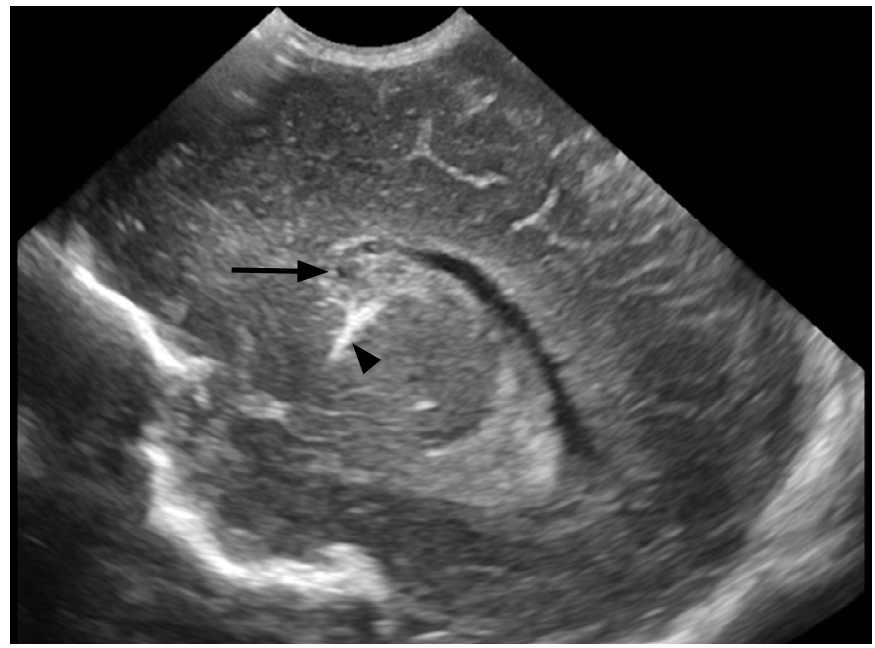

B

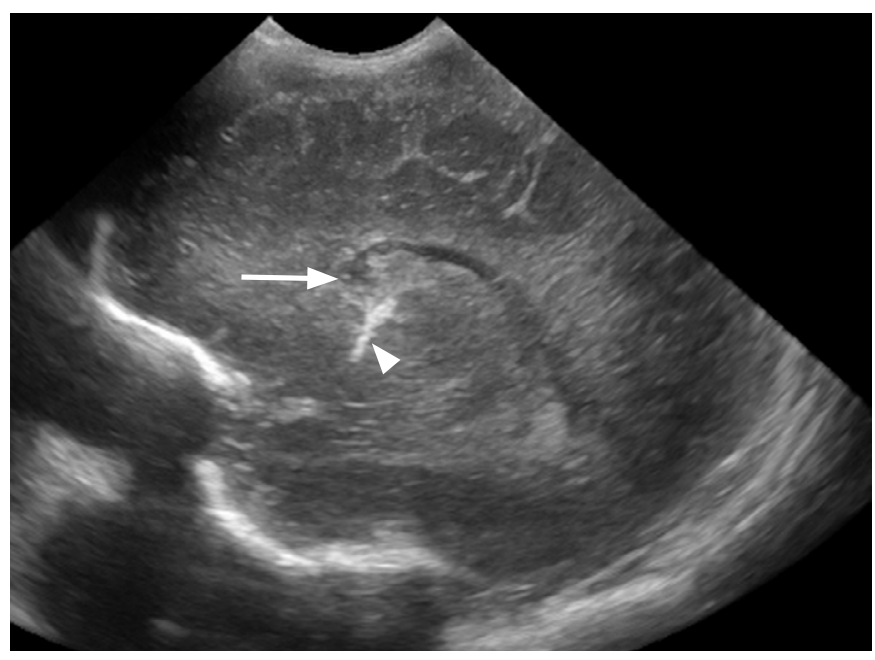

D

Fig. 3. A 32-day-old preterm infant with multifocal infarctions and lenticulostriate vasculopathy.

A-D. The 2-dimensional cranial ultrasonography (2DUS) images (A, B) and cranial 3-dimensional cranial ultrasonography (3DUS) captured images (C, D) are shown. Clear focal infarction with cystic change in left frontal periventricular white matter and bilateral basal ganglia (arrows in A and B) and lenticulostriate vasculopathy in the left basal ganglia (arrowhead in B) is depicted on 2DUS. The 3DUS scan shows equivalent image quality and focal infarction with cystic change in the left frontal periventricular white matter and bilateral basal ganglia (arrows in $C$ and D), lenticulostriate vasculopathy in the left basal ganglia (arrowhead in D), consistent with the findings obtained by 2DUS. The cine clip of 3DUS scan is presented as Video clip 3.

Table 3. Diagnostic confidence of 2DUS and 3DUS

\begin{tabular}{|c|c|c|c|c|c|c|}
\hline & \multicolumn{3}{|c|}{ Reviewer 1} & \multicolumn{3}{|c|}{ Reviewer 2} \\
\hline & 2DUS & 3DUS & P-value & 2DUS & 3DUS & P-value \\
\hline GMH & $2.2 \pm 0.4$ & $3 \pm 0$ & $<0.001$ & $2.2 \pm 0.5$ & $3 \pm 0$ & $<0.001$ \\
\hline PVE & $2.8 \pm 0.4$ & $2.8 \pm 0.4$ & 0.637 & $2.6 \pm 0.5$ & $2.8 \pm 0.4$ & 0.020 \\
\hline VM & 3 & 3 & - & 3 & 3 & - \\
\hline
\end{tabular}

2DUS, 2-dimensional cranial ultrasonography; 3DUS, 3-dimensional cranial ultrasonography; GMH, germinal matrix hemorrhage; IVH, intraventricular hemorrhage; $\mathrm{PVE}_{\text {, }}$ periventricular echogenicity; VM, ventriculomegaly; $\mathrm{FL}$, focal parenchymal lesion. 
significantly shorter than 2DUS $(\mathrm{P}<0.001)$.

\section{Discussion}

In the context of clinically relevant issues in neonatal cranial US, such as hemorrhage, ventriculomegaly, and focal parenchymal abnormalities, our study showed that 2DUS and 3DUS demonstrated similar performance and that 3DUS was associated with a higher confidence level than traditional US findings. US is operatordependent, and the probe or sonic window can be changed on a case-by-case basis by the expert making the diagnosis during the scan. However, in clinical settings, sonograms routinely require reinterpretation by radiologists with more specialization in the field than the operator. This is particularly relevant for some countries where US technicians obtain the images, or if US is performed by non-specialist radiologists in high-volume hospitals carrying a heavy workload. In addition, follow-up requires the review of previous sonograms. In such a situation, it can be difficult to confirm abnormalities seen in scan images that are based on limited numbers of captured 2DUS images. In contrast, cine images obtained from 3DUS can be recalled at any time for study, enabling the entire brain region to be screened, which increases the interpreter's confidence level.

Furthermore, the 3DUS technique requires a significantly shorter time for scanning than 2DUS. Our study used a 3D volume transducer that mechanically sweeps the transducer array. Compared to manual cine sweeps, mechanically swept 3DUS produces smooth cine images that can be acquired in a short time period of 2.2 seconds, whereas manual cine sweeps were shown to be acquired after 6 seconds [16]. In our study, the image quality of 3D cine US did not appear to be greatly affected due to the short scan time of 2.2 seconds per sweep and the high temporal resolution of the cine clip (47 frames per second). From an operator's perspective, we can conclude that mechanically-swept 3D US is associated with a reduced workload in performing scans. Stenman et al. [3,4] noted the observer dependency in US and the increased workload of radiologists given the growing demand for US studies, and suggested obtaining cine clips as a potential new method that could resolve these issues. The results of the present study are in close agreement with those reports in the context of maintaining diagnostic equivalency with a significant reduction in imaging time and, consequently, labor. In addition, the majority of patients (58\%) had no abnormality, meaning that the use of 3DUS as a screening tool by non-specialist radiologists is also expected. Moreover, neonatal care requires minimal handling by medical staff; therefore, reducing the scan time is important for neonates and young infants [17].

O'Dell et al. [16] recently reported that cine imaging alone was equivalent to combined static and manually obtained cine imaging in cranial US screening in premature infants. In their study, intraventricular and parenchymal hemorrhages were detected equally well with both methods, while cine imaging alone overestimated periventricular leukomalacia and missed few small choroid plexus cysts in comparison with dual static and cine image review. Our study design is different from that of O'Dell et al. [16], in that we directly compared 2D static US and 3D cine images of the brain, and the 3D cine images were obtained with a mechanically swept 3D US probe. As previously discussed, a mechanically swept $3 \mathrm{D}$ probe is associated with faster scanning, allows more constant and smooth cine images, and requires less operator experience than manual sweeps. Therefore, our study resulted in better concordance between the static and cine images.

Some limitations and unexpected aspects of our study must be addressed. First, there is insufficient evidence to conclude that a disparity exists between the two techniques in view of the small sample size. Second, both 2DUS and 3DUS scanned images were solely obtained by a convex transducer with lower resolution than a linear transducer. Third, both 2DUS and 3DUS scans were conducted only through the anterior fontanelle, implying that infratentorial abnormalities, including cerebellar hemorrhage and subdural hemorrhage through the mastoid fontanelle and small IVH through the posterior fontanelle, were prone to be missed. However, a volume scan through the mastoid fontanelle is also feasible. Fourth, the use of a relatively large 3D probe in a neonate's small anterior fontanelle may limit the field of view of 3DUS volume scanning. However, there was no statistically significant difference in image quality between 2DUS and 3DUS in our study. Finally, head motion by the subjects during the scan could have affected the readings. Although there was a small amount of head motion during 3DUS scanning, the present study did not evaluate the degree of motion during the scan, as the blurring in the procured images was within the acceptable image quality. This can be explained by the fact that the brain parenchyma was captured in the designated wide field of view of 3DUS despite a tilted axis.

In conclusion, regarding the reinterpretation of cranial US, 3DUS showed very high concordance with 2DUS and a similar image quality. 3DUS also increased diagnostic confidence for several image findings and significantly decreased the scan time.

ORCID: Yu Jin Kim: http://orcid.org/0000-0001-9230-6477; Young Hun Choi: http:// orcid.org/0000-0002-1842-9062; Hyun Hae Cho: http://orcid.org/0000-0002-48652601; So Mi Lee: http://orcid.org/0000-0002-2073-8198; Ji Eun Park: http://orcid. org/0000-0003-1305-0931; Jung-Eun Cheon: http://orcid.org/0000-0003-14792064; Woo Sun Kim: http://orcid.org/0000-0003-2184-1311; In-One Kim: http:// orcid.org/0000-0001-5800-3536 


\section{Conflict of Interest}

No potential conflict of interest relevant to this article was reported.

\section{Supplementary Material}

Video clip 1. A 30-day-old preterm infant with germinal matrix hemorrhage (GMH) and abnormal periventricular white matter. Left small GMH was noted at 3-dimensional cranial ultrasonography (3DUS). Heterogeneous periventricular white matter echo at left frontoparietal white matter is shown at 3DUS. This is one discordant case on 3DUS between the two reviewers; reviewer 1 scored abnormal periventricular echogenicity (PVE) on 2-dimensional cranial ultrasonography (2DUS) but borderline PVE on 3DUS, reviewer 2 scored abnormal PVE on both 2DUS and 3DUS. It was later diagnosed as a non-cavitary white matter injury on follow-up magnetic resonance imaging (https://doi.org/10.14366/usg.17009.v001).

Video clip 2. A 34-day-old preterm infant with intraventricular hemorrhage (IVH). The 3-dimensional cranial ultrasonography scan shows small IVH in right lateral ventricle clearly more than 2-dimensional cranial ultrasonography (https://doi.org/10.14366/ usg. 17009.v002).

Video clip 3. A 32-day-old preterm infant with multifocal infarctions and lenticulostriate vasculopathy. The 3-dimensional cranial ultrasonography scan shows equivalent image quality and multifocal infarction with cystic change in the bilateral frontal periventricular white matter, bilateral basal ganglia, and the right germinal matrix hemorrhage, consistent with the findings obtained by 2-dimensional cranial ultrasonography (https://doi.org/10.14366/usg.17009.v003).

\section{References}

1. American Institute of Ultrasound in Medicine (AIUM); American College of Radiology (ACR); Society of Radiologists in Ultrasound (SRU). AIUM practice guideline for the performance of neurosonography in neonates and infants. J Ultrasound Med 2014;33:1103-1110.

2. Riccabona M, Nelson TR, Weitzer C, Resch B, Pretorius DP. Potential of three-dimensional ultrasound in neonatal and paediatric neurosonography. Eur Radiol 2003;13:2082-2093.

3. Stenman C, Jamil S, Thorelius L, Knutsson A, Smedby O. Do radiologists agree on findings in radiographer-acquired sonographic examinations? J Ultrasound Med 2013;32:513-518.

4. Stenman C, Thorelius L, Knutsson A, Smedby O. Radiographer- acquired and radiologist-reviewed ultrasound examination: agreement with radiologist's bedside evaluation. Acta Radiol 2011;52:70-74.

5. Downey DB, Fenster A, Williams JC. Clinical utility of threedimensional US. Radiographics 2000;20:559-571.

6. Kim SH, Choi Bl. Three-dimensional and four-dimensional ultrasound: techniques and abdominal applications. J Med Ultrasound 2007;15:228-242.

7. You SH, Suh SI, Ryoo IS, Son GR, Lee YH, Seo HS, et al. Assessing the accuracy and reliability of ultrasonographic three-dimensional parathyroid volume measurement in a patient with secondary hyperparathyroidism: a comparison with the two-dimensional conventional method. Ultrasonography 2017;36:17-24.

8. Fenster A, Downey DB, Cardinal HN. Three-dimensional ultrasound imaging. Phys Med Biol 2001;46:R67-R99.

9. Ong CL. The current status of three-dimensional ultrasonography in gynaecology. Ultrasonography 2016;35:13-24.

10. Khurana A, Dahiya N. 3D and 4D ultrasound: a text and atlas. New Delhi: Jaypee Brothers Medical Publishers, 2004.

11. Lowe LH, Bailey Z. State-of-the-art cranial sonography: Part 1, modern techniques and image interpretation. AJR Am J Roentgenol 2011;196:1028-1033.

12. Siegel MJ. Pediatric sonography. Philadelphia, PA: Wolters Kluwer Health/Lippincott Williams \& Wilkins, 2011.

13. GE Healthcare. Voluson E8 Expert datasheet [Internet]. Boston, MA: General Electric Company, 2002 [cited 2017 Apr 5]. Available from: http://www3.gehealthcare.com/en/products/categories/ultrasound/ ultrasound_probes\#tabs/tabDDE2A62CD23946C8A4BB893C46D6 CD79.

14. van Wezel-Meijler G, van der Knaap MS, Sie LT, Oosting J, van Amerongen $\mathrm{AH}$, Cranendonk $\mathrm{A}$, et al. Magnetic resonance imaging of the brain in premature infants during the neonatal period. Normal phenomena and reflection of mild ultrasound abnormalities. Neuropediatrics 1998;29:89-96.

15. Lee JH, Choi YH, Cheon JE, Lee SM, Cho HH, Shin SM, et al. Improved abdominal MRI in non-breath-holding children using a radial k-space sampling technique. Pediatr Radiol 2015;45:840846.

16. O'Dell MC, Cassady C, Logsdon G, Varich L. Cinegraphic versus combined static and cinegraphic imaging for initial cranial ultrasound acreening in premature infants. Pediatr Radiol 2015;45:1706-1711.

17. Bada HS, Korones SB, Perry EH, Arheart KL, Pourcyrous $M$, Runyan JW $3 r d$, et al. Frequent handling in the neonatal intensive care unit and intraventricular hemorrhage. J Pediatr 1990;117(1 Pt 1):126-131. 